\title{
Derek Mahon, La Mer hivernale et autres poèmes
}

\section{Claude Fierobe}

\section{OpenEdition}

1 Journals

\section{Electronic version}

URL: http://journals.openedition.org/etudesirlandaises/3854

DOI: 10.4000/etudesirlandaises.3854

ISSN: 2259-8863

\section{Publisher}

Presses universitaires de Caen

\section{Printed version}

Date of publication: 30 June 2014

Number of pages: $227-228$

ISBN: 978-2-7535-3449-0

ISSN: 0183-973X

\section{Electronic reference}

Claude Fierobe, «Derek Mahon, La Mer hivernale et autres poèmes », Études irlandaises [Online], 39-1 | 2014, Online since 30 June 2016, connection on 22 September 2020. URL : http://

journals.openedition.org/etudesirlandaises/3854; DOI : https://doi.org/10.4000/etudesirlandaises. 3854

\section{(c) (i) (2)(2)}

Études irlandaises est mise à disposition selon les termes de la Licence Creative Commons Attribution - Pas d'Utilisation Commerciale - Partage dans les Mêmes Conditions 4.0 International. 
The title poem, "Fuíoll Feá" [Woodshavings] (114/5) is not perhaps the most captivating poem of the collection in poetic terms. However the poem is central to the poet's identity (he is after all a carpenter's son) and his enterprise. In the poem "Ailm" [Elm] (116/7) addressed to his fellow poet Tomás MacSiomóin the intertext is clearly "Cill Chais" the great poem of lament for the end of the Gaelic Age where the felled trees are symbolic also of the thinning out of the language. Images of replanting and trees abound in Ó Muirthile's work. He describes the poetic life in the following terms:

\author{
Throimaigh ár mbeo \\ thriomaigh ár ngui: \\ imionn ár n-arán \\ laethuil ina bhrus \\ smiota ar na liopaí. \\ [Our life has come to dust, \\ our prayers: \\ our daily bread \\ falls from our lips \\ in shattered crumbs.]
}

The paronomasia at play between "ár ndán" (our poem) in the following verse and "ár n-arán" (our bread) in this verse leaves us no illusions as to the loneliness and frustration of Ó Muirthile and his fellow Irish language poets (an explanation perhaps of the earlier paronomasia of the initial poem between "meirg" and "mairg", rust and regret); they are frequently castigated in the Irish media as both irrelevant and inferior. This wonderful book proves those naysayers wrong on both counts.

Clíona Ní RíORDÁin

Université Sorbonne Nouvelle-Paris 3

Derek Mahon, La Mer hivernale et autres poèmes, traduit de l'anglais (Irlande) et préfacé par Jacques Chuto, Édition bilingue, Le Chambon-sur-Lignon, Cheyne éditeur, coll. « D’une voix l'autre », 2013, 172 p., ISBN 978-2-84116193-5, $24 €$.

Ce recueil rassemble des textes choisis dans les Collected Poems de 1999, mais les révisions opérées pour les New Collected Poems de 2011 ont été prises en compte par 
le traducteur : quarante-huit poèmes choisis de manière à refléter les préoccupations majeures d'un homme qui " n'aime guère son époque " et s'en prend aux travers du monde contemporain. Il dénonce la défiguration de l'Irlande, depuis le massacre du milieu naturel (déforestation de l'Ulster au XviI ${ }^{\mathrm{e}}$ siècle dans " Going Home »), jusqu'à l'invasion tonitruante des cars de touristes " grotesques en casquette de baseball et survêt en nylon » ("Night Thoughts ", extrait d'une suite justement nommée «Decadence »). Le poids du passé, Pompéi, Treblinka, des « Troubles » ou encore de la guerre civile ne peut être évacué, et c'est la tâche du poète que de parler au nom de tous "les suppliants sans voix " ("A Disused Shed in Co. Wexford"). Il fustige un mode de vie où le heavy metal le rend "fou de rage " tandis qu'il s'accroche à ses livres, " ces trucs périmés » ("Rock Music »), ou à la beauté de la nature. L'uniformité sans âme a tout envahi. Avec «les Dieux bannis " Mahon rêve d'un monde « sans ordinateurs ni voitures/Ni cieux nucléaires,/Où la pensée, c'est caresser des pierres,/Et la sagesse, un moment de silence quand se lève la lune. » On le voit se raidir pour dépasser l'amertume, la nostalgie et le désespoir (l'émouvant "Dawn at St Patrick's »), et s'en remettre aux « Consolations de la philosophie " pour " vivre la vie que nous aurions pu vivre " sans exclure qu'un jour l'humanité prenne un nouveau départ. Jacques Chuto sait ce que traduire veut dire : il a déjà traduit Mahon (traducteur lui-même) en 1991, et fréquenté longuement un certain J.-C. Mangan expert en la matière... Décidé à s’en tenir « à la fidélité sans renoncer à la beauté ", entouré des nombreux conseils de l'auteur auquel il rend hommage, il s'y entend à maintenir une forme strophique virtuose ("The Hunt by Night "), à trouver des rimes en écho à l'original sans forcer le sens (" Derry Morning »), et surtout à imprimer à ses traductions le rythme qui serre au plus près celui de la voix du poète : "La Mer hivernale ", credo poétique de Mahon, heureusement choisi pour donner son titre au recueil, est à cet égard une belle réussite où la " litanie des toponymes " chère à John Montague, en mêlant l'Irlande et la Grèce, invite à « Repartir à zéro, de l'ancien faire/Un renouveau. » Ce recueil est bilingue : en comparant l'original et la version française, le lecteur pourra saisir par quels procédés délicats est rendue la complexité de l'œuvre. Mahon rend hommage aux écrivains chers à son cœur, cités nommément ou perçus en filigrane dans la trame des textes : Ovide, Pindare, Swift, J.G. Farrell, McNeice, Heaney, Baudelaire, Camus, et beaucoup d'autres. Les notes suffisantes et précises éclairent les points obscurs. Ce livre élégant est un bel objet qui ajoute au plaisir de la double lecture. Qu'on ne se méprenne pas : la critique véhémente de Mahon à l'égard du monde qui l'entoure est avant tout celle d'un poète de première grandeur. Ici, il a trouvé son traducteur.

Claude Fierobe Université de Reims Champagne-Ardenne 